\title{
Simulating the Allocation of Organs for Transplantation
}

\author{
DAVID THOMPSON* ${ }^{*}$ and LARRY WAISANEN \\ Altarum Institute, Ann Arbor, 3520 Green Court, MI 48105, USA \\ E-mail: david.thompson@altarum.org \\ ROBERT WOLFE \\ Scientific Registry of Transplant Recipients, Kidney Epidemiology and Cost Center, Department of Biostatistics, University of Michigan, Ann Arbor, MI, \\ USA \\ ROBERT M. MERION \\ Scientific Registry of Transplant Recipients, Division of Transplantation, Department of Surgery, University of Michigan Medical School, Ann Arbor, MI, \\ $U S A$ \\ KEITH MCCULLOUGH and ANN RODGERS \\ University Renal Research and Education Association, Ann Arbor, MI, USA
}

\begin{abstract}
The demand for donated organs greatly exceeds supply and many candidates die awaiting transplantation. Policies for allocating deceased donor organs may address equity of access and medical efficacy, but typically must be implemented with incomplete information. Simulation-based analysis can inform the policy process by predicting the likely effects of alternative policies on a wide variety of outcomes of interest. This paper describes a family of simulations developed by the US Scientific Registry of Transplant Recipients and initial experience in the application of one member of this family, the Liver Simulated Allocation Model (LSAM).
\end{abstract}

Keywords: transplantation, simulation, organ allocation, organ distribution, end-stage liver disease, liver transplant

\section{Introduction}

\subsection{Scarcity of organs for transplantation}

Allocating the scarce and precious resource of donated organs in an equitable, effective, and efficient manner is a complex problem. Policies are the subject of intense discussion, and disagreement is inevitable, because the award of an organ to one candidate denies it to others. It is critical to inform policy deliberations with valid and objective information. Predicting the impacts of proposed new organ allocation policies is very difficult, because there are many factors that can change in unpredictable ways when rules change. The types of candidates on waitlists and the medical statuses of candidates and graft recipients change over time; large numbers of patients and organs are involved; patient and organ characteristics are diverse; medical outcomes cannot be predicted with certainty; and policies can produce results that may not be anticipated. Simulation offers a practical way to represent this dynamic system and to provide information needed for informed debate before a policy change is put into effect. This paper describes a family of simulations developed for this purpose and initial experience with one member, the Liver Simulated Allocation Model (LSAM), in the analysis of liver allocation policies.

The importance of the problem is illustrated by the example of liver transplantation. For many persons with end-stage

* Corresponding author. liver disease, a liver transplant may be the only effective therapy, but every year many die because too few donor livers are made available. In order to receive a cadaveric transplant, candidates must register on a national waitlist and wait for an organ to become available and be allocated to them. During 2001, 1970 people died while waiting for a liver transplant in the United States, while 4663 cadaveric transplants and 514 transplants from living donors were performed. The number of persons on the waitlist grew $12 \%$ to 18,214 at the end of 2001 [1]. At least 140 others died after being removed from the waitlist because they were no longer healthy enough to survive or benefit from a transplant or for other reasons. Although liver transplantation carries some risks, over $70 \%$ of transplant recipients (under age 65) are still alive 5 years after liver transplantation [2].

\subsection{The U.S. organ allocation system}

With oversight and funding from the Department of Health and Human Services (HHS), the Organ Procurement and Transplantation Network (OPTN) proposes policies for organ allocation, maintains the waitlists, implements the policies, and collects data on transplantation. Transplant centers at hospitals around the United States perform transplants with organs procured from cadaveric donors by local organ procurement organizations (OPOs). Patients in need of transplants are entered onto the waitlist. When an organ becomes available, the active candidates on the waitlist are ordered as 
specified by the current policy and the liver is offered to candidates in this order. The organ is transplanted to the first candidate to accept the offer, and that patient is then removed from the waitlist. Candidates are classified according to risk of short-term mortality on the waitlist, with priority given to those at highest risk. Methods of risk measurement have varied historically as different policies have been in effect.

Specifics of allocation policies vary among different organs. In the allocation of livers, for example, geography also plays an important role. In general, ordering of candidate recipients of a given donor organ occurs within three nested sets of geographic area. Organs are first offered to candidates who are within the service area of the donor hospital's organ procurement organization (OPO). Next, candidates within a larger, usually multiple state region are considered, and then finally the remainder of candidates in the rest of the United States.

In February 2002, the prioritization system for non-status 1 candidates was changed from one based upon waiting time within these broad categories of medical necessity to one based on a scaled measure of the 90-day risk of mortality while on the waiting list [3]. This change was motivated by the reality of there being more patients than organs - a waiting-time based system will generally result in the allocation of organs to those patients who are healthy enough to survive until they can move to the front of the waiting-time queue. Thus, a waiting time based system fails to prevent deaths among those at highest risk for death.

In this paper, we describe a family of simulation models that includes the liver model and compare results to those that actually occurred when the policy change was implemented. Previous simulation-based studies of liver allocation policies include Howard's [4] analysis of sickest-patient-first policies, a model of the liver allocation system in the United Kingdom developed by Ratliffe et al. [5], and the United Network for Organ Sharing (UNOS) Liver Allocation Model (ULAM ${ }^{\mathrm{SM}}$ ), developed and applied when UNOS was the contractor operating the U.S. Scientific Registry of Transplant Recipients (SRTR) [6-11].

\subsection{Simulation-based analyses of organ allocation policies}

The organ transplantation community faces a wide variety of issues that can be addressed by simulation-based analyses. Further, the prospect is that more issues will arise in the future, as medical science and the demographics of the population change. In response to this need, the Health Resources and Services Administration (HRSA) of HHS decided to acquire a new set of simulation tools for analysis of issues in liver, heart, lung, kidney, and pancreas transplant allocation policies. There was a broad need in the transplant field to develop new allocation simulations that would:

- let investigators easily explore a wide range of policies;

- allow the simulation program to be easily rewritten and, to some extent, let users define new policies without new programming;
- allow several of the most detailed simulation assumptions to be modeled externally to the program and be used as input to the program;

- be validated against historical and future data;

- be coded in short time and not require expensive or proprietary simulation software packages; and

- be available to other researchers in the organ transplant community.

A family of three simulations was designed by the University Renal Research and Education Association (URREA) with the University of Michigan, under its contract as the current contractor for the SRTR to meet these requirements. LSAM models the allocation of livers; the Thoracic Simulated Allocation Model (TSAM) is an integrated heart and lung allocation model; and the Kidney-Pancreas Simulated Allocation Model (KPSAM) models the allocation of kidneys and pancreata. The simulations are written in Object Pascal, using Borland's Delphi ${ }^{\mathrm{TM}}$ development environment. The resulting code is portable and openly available.

\section{Modeling methods}

\subsection{General approach}

The models separate two components of the organ transplantation system. One component is the environment in which an allocation policy operates, which is defined by the stream of patients who are on and who are added to the waitlist and by the stream of organs that are available for transplant. A second component consists of the policies that link the patients to the organs that are available and the subsequent experience of transplant recipients. These two components are logically separable. The environment is addressed principally as an input stream to the program while the policies are largely incorporated directly into the code of the program. Very broad and flexible input specifications for the environment data are allowed and very flexible families of policies can be implemented within the framework of the programs, as described below.

The separation between environment and allocation system is reflected in the model structure shown in figure 1. Arrivals of patients, organs, and patient status changes are generated in $\mathrm{SAS}^{\mathrm{TM}}$ and input to the allocation system model. The general approach to modeling the allocation system is Monte Carlo, event-sequenced simulation, coded in Object Pascal. This model generates sequences of pseudo-random numbers to generate realizations of random processes (e.g., organ acceptance decisions and graft survival times), and advances time by sequencing discrete events in time order.

\subsection{Candidate and organ arrivals}

As shown in figure 1, each simulation has been designed to take advantage of characteristic structures of the organ transplantation and allocation system. Certain processes operate independently of the rest of the system, because there is 


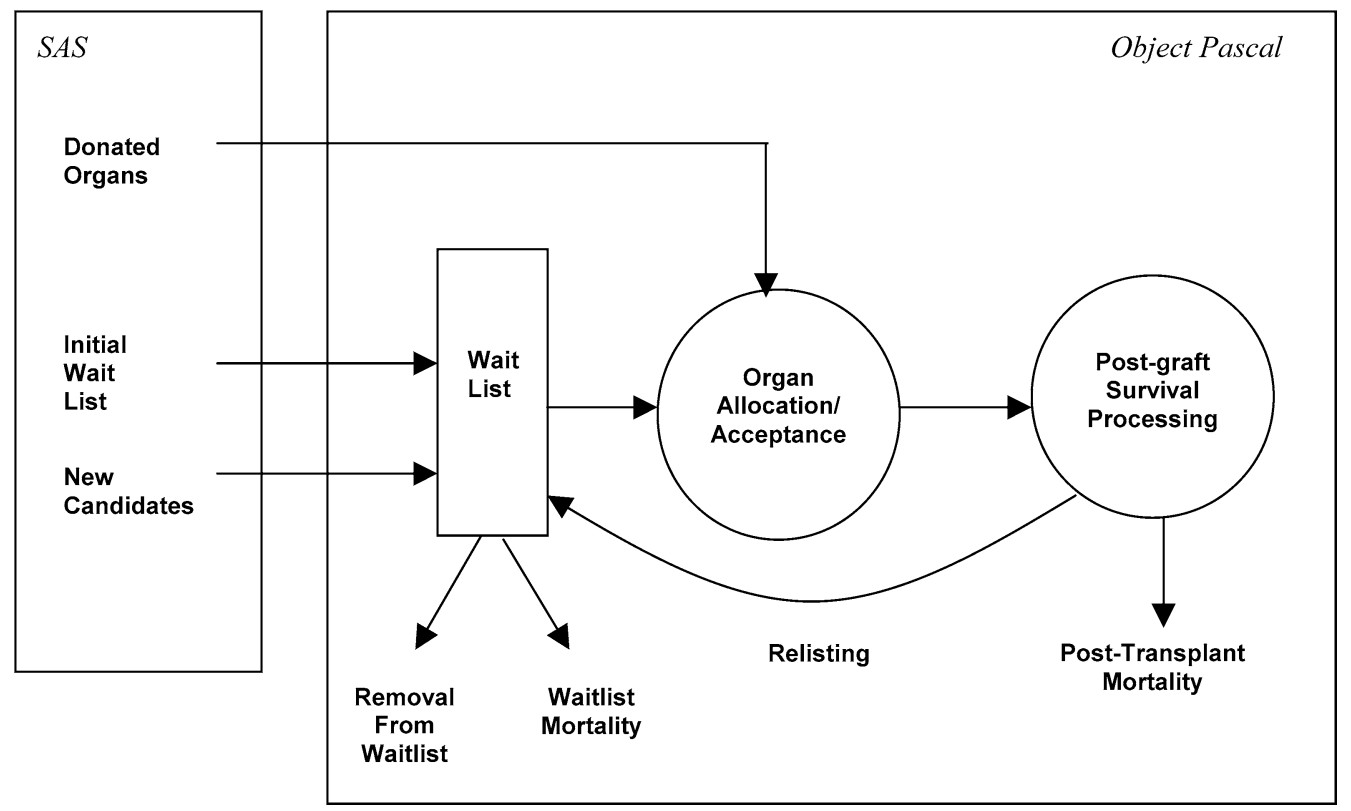

Figure 1. Top-level schematic of patient and organ flows.

no feedback to them. Two of these are the donation of organs and the listing of candidates for their first transplants. These processes are represented as streams of arrivals input to the simulation. Model users can generate arrival streams from historical arrival data, synthetic data, reordered historical data, or combinations of these methods. In practice, we use $\mathrm{SAS}^{\mathrm{TM}}$ to prepare input arrival records from historical and synthetic arrival data. This allows a wide variety of environments to be evaluated with a wide variety of data sources. The initial waitlist membership (i.e., at the starting simulated time) is also input to the model. Arrival records specify characteristics of candidates at the time of listing (age, transplant center, etc.) and characteristics of donors and organs (blood type, weight, etc.).

\subsection{Candidate status}

A time-ordered input stream describes changes to each candidate's medical conditions and listing statuses that would occur if the candidate received no transplant. Candidates' medical conditions may improve or deteriorate over time, and a candidate may die, leave the system, become temporarily inactive, or resume active status prior to receiving a transplant. Model users may define new patient attributes and status codes, or use historical defaults. Patient histories can be structured to include covariates used in survival and organ acceptance models, such as organ-specific medical status codes.

Because the time of graft offer cannot be predicted prior to running the simulation, each candidate's status stream describes the candidate's entire history from the time of listing until either death or the end of the simulated time period, whichever comes first. Users can generate the status streams synthetically or use actual historical statuses (although the histories of actual candidates who received transplants need to be synthetically extended to either the predicted time of death on the waitlist or, in the absence of a transplant, the end of the simulated time horizon). In practice, we use $\mathrm{SAS}^{\mathrm{TM}}$ to generate status change input records from historical and synthetic bases of status change data.

Provision is also made for candidates whose transplanted organs fail and who rejoin the waitlist. A candidate's original input stream of status changes ceases to be relevant when the simulation awards a graft to that candidate. For this reason, the input stream cannot be applied to a patient who receives a graft and later rejoins the waitlist because of a failing graft. Instead, separate pools of status histories can be generated or provided to the simulation, from which the simulation samples a history to assign to the relisted patient.

\subsection{Organ allocation}

A time ordered input stream provides the arrival time and characteristics of donated organs. When an organ becomes available, the simulation reorders the active candidates on the waitlist according to the operative policy and offers the organ to candidates in this order. Because donor and candidate characteristics (e.g., blood type compatibility) are compared, the waitlist is reordered every time a new organ is donated (the "match process"). The organ is transplanted to the first candidate to accept the offer.

\subsection{Organ acceptance and post-graft survival}

To allow for future modeling improvements, the simulations provide flexible representations of the organ acceptance decision and post-graft processes. Simulation users may define models for such processes in terms of donor-candidate categories (strata), vectors of donor and candidate variables $(X)$, and a vector of coefficients of these variables $\left(B_{j}\right)$ for each stratum $(j)$. Further flexibility is offered by allowing the de- 
finition of other inputs to the process models as simple algebraic functions of these original inputs and of the status history characteristics, which can change over time.

The probability of organ acceptance is modeled as a logistic function of the inner product $X B_{j}$. This functional form provides considerable flexibility for representing acceptance probabilities and their dependence on donor and organ characteristics. This approach allows flexibility of application to each organ model. The available covariates could be explored to find a combination that best explains historical acceptance decisions. For the acceptance decision, the model samples a pseudo-random number and compares it to an acceptance probability computed as a logistic function of candidate and donor characteristics.

Acceptance of an organ leads to transplantation and removal of the recipient from the waitlist. The simulation then tracks the post-graft experience of the recipient, including the potential for graft failure and rejoining the waitlist, and mortality.

Post-graft survival is represented by a Cox proportional risk model or a Weibull model. For the Cox proportional risk model, the user also supplies a baseline survival function in tabular form for each stratum. Specific covariates in the survival function depend on the organ being modeled, but include a variety of pre-defined and user-defined characteristics of patient and organ, including measures of clinical condition of the patient at the time of transplant. The simulation generates a survival time by sampling a pseudo-random number and inverting the survival function. Death may be due to organ failure. In LSAM and TSAM, death occurs within a few days of organ failure unless a second transplant occurs first. In KPSAM, there is also the possibility that the patient will return to dialysis, which is an alternative medical treatment for kidney failure.

Patients are randomly allowed to relist prior to the sampled death date. The probability and time of relisting, stratified by post-graft survival time, are input to the model. The simulation adds post-graft status change events, including death or removal from the waitlist, to the list of scheduled events.

\subsection{Model boundaries}

It is important to recognize that the representation of the transplantation system does not stop strictly with the software, but also includes the assumptions used in preparing inputs to the model. This data-centered approach is prudent, given the level of uncertainty and changing knowledge about the nature of many of the constituent biological and behavioral processes. However, this approach places a number of important process representations outside the boundaries of the code and in the hands of those who prepare the input data, defined in the User Manual [12]. This data-centered approach provides the simulations with considerable flexibility, but it also requires of users a level of acquaintance with details of the simulation and its input data. Since future policies are likely to involve unforeseeable clinical status measures, it is not practical to attempt to generate all of the potential types of complex stochastic status histories within the simulation program. The waitlist status histories were left outside of the simulation programs, resulting in great flexibility for user specification of these histories.

\section{Representing policies}

All simulations of the family incorporate a policy description language by which users may specify a wide variety of alternative allocation policies without reprogramming the simulation. Users may describe allocation policies in text data files and further edit and save these policies via the simulation's graphical user interface. The function of the policy description language is to specify an algorithm for ordering the waitlist, given a donated organ of specified characteristics, in terms of:

- criteria for screening out potential candidates for lack of suitability (e.g., unacceptable body weight of the liver donor);

- grouping candidates into priority classes based on common characteristics (e.g., medical urgency codes and location relative to the donor's OPO);

- ranking candidates within a classification based on sequential sorts and additive point awards (e.g., waiting time or mortality risk score); and

- defining subgroups of priority classes (e.g., giving pediatric candidates priority for a pediatric organ, or partitioning based on a threshold value of mortality risk).

These features can represent current and historical policies for heart, lung, liver, kidney, and pancreas, as well as a wide variety of alternative policies. Flexibility is enhanced by the ability to add new, user-defined fields to input records (i.e., new characteristics of candidates and organs) and reference these fields in the definition of an allocation policy. Userdefined input fields are also included in the vector of donor and candidate variables in the organ acceptance and post-graft survival models.

Table 1 lists an extract from an example liver allocation policy. A policy may be divided into sections that apply to different groups of donors, such as the example shown here for adult donors. Within each donor group, the policy lists categories of candidates in priority order. In this example, the top priority category consists of candidates in medical status 1 (the most urgent cases) who are located in the same local unit as the donor. These candidates (if any) are ranked in order of allocation points, status 1 waiting time, and total waiting time. (Examples of factors that can be considered in allocating points are given in section 4.) If no candidate in this category accepts the liver, the model proceeds to the next line in the policy definition. This is only an example of the criteria that can be used for screening and ranking. 
Table 1

Extract from an LSAM policy definition file. ${ }^{\mathrm{a}}$

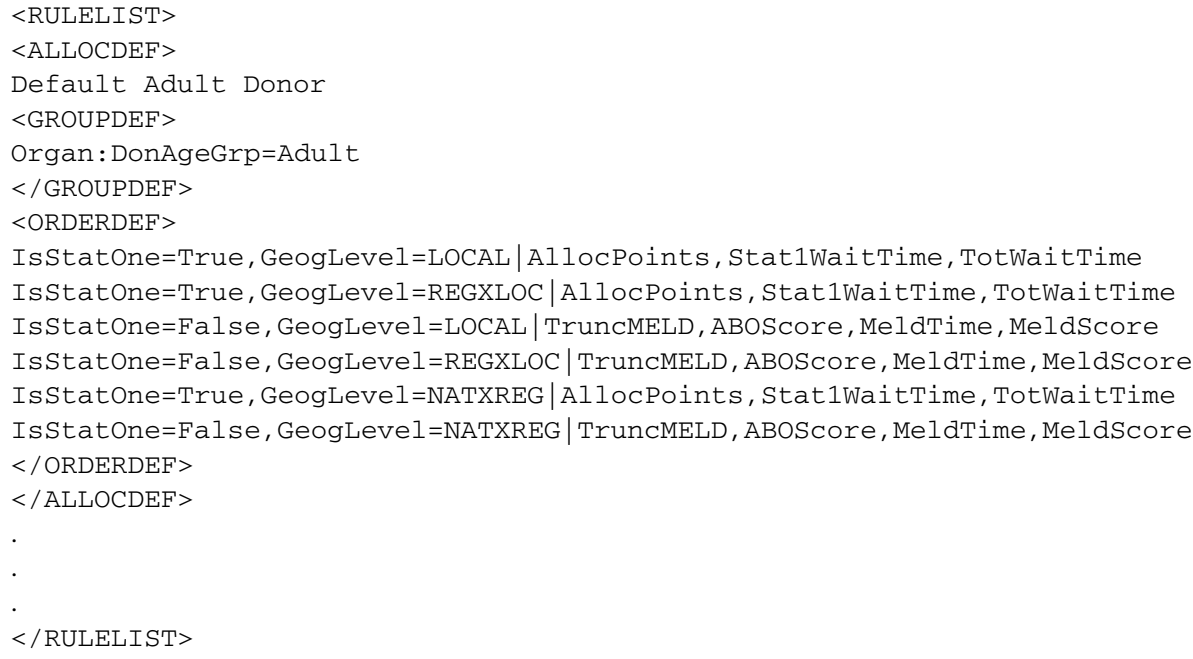

${ }^{a}$ This table demonstrates that prioritization can be based on broad ordered categories with continuous scales within each category that are specific to that category. Note that waiting time is used to rank order status 1 candidates, while MELD score is used to prioritize non-status 1 patients.

\section{Initial application of LSAM}

LSAM development began in February 2001, and a prototype was completed in June 2001. In September 2001, LSAM version 0.9.6 (a version with "hard coded" allocation rules) was validated against historical OPTN data and applied to a prospective analysis of a proposed change to the OPTN liver allocation policy incorporating the Model for End-stage Liver Disease (MELD) disease severity index.

\subsection{Current and previous policies}

Until recently, medical urgency was measured through a status-based set of medical urgency codes. In the most recent version of this system, patients with rapid and life-threatening liver dysfunction were classified as status 1 , as were transplant recipients whose graft failed within seven days. Patients with chronic liver disease were classified into statuses 2A, 2B, and 3 , in decreasing order of severity and based largely on the Child-Turcotte-Pugh (CTP) scale [13]. The CTP scale combines the international normalized ratio (INR, a measure of the blood's ability to clot), the bilirubin level in the blood (a measure of liver function), the albumin level in the blood (a measure of nutrition), the presence of encephalopathy, and the presence of ascites (fluid in the abdomen). The statusbased codes also recognized patients who were temporarily inactive (status 7), patients who died while waiting for a transplant (status 8), and patients who had been removed from the waiting list (status 9).

MELD is a disease severity index, based on easily obtained measurements of patients, which has been shown to be a reliable measure of mortality risk for end-stage liver disease $[14,15]$. MELD eliminates the two subjective factors of the CTP scale (encephalopathy and ascites), retains the INR and bilirubin, and adds the creatinine level in the blood (a measure of kidney function). MELD is computed by a regression formula as a function of a candidate's INR, bilirubin, and creatinine levels.

On February 27, 2002, the OPTN liver allocation system started operation of a new liver allocation policy that eliminated the former medical statuses for candidates with chronic disease and substituted disease severity with MELD scores [3]. Status 1 was retained as a classification for acutely ill candidates, and all other adult patients were to be assigned MELD scores, as measures of the three-month risk of mortality of remaining on the waitlist. A related risk measure, the Pediatric End-stage Liver Disease (PELD) score [16], was created for pediatric candidates.

\subsection{Data used}

The simulation runs used actual data for all patients on the waitlist in 1999, and simulated all activity in the period from $1 / 1 / 99$ through 12/31/99. We input the actual entire history of status changes and death dates for all patients, except for those who received a transplant. The MELD-based runs required histories of MELD changes for all patients. These were selected randomly from actual histories of patients in a University of Michigan study of MELD mortality [15], subject to matching initial waitlist status and survival time. Since the simulation does not give transplants to all patients who were actually transplanted, it was also necessary to create artificial status change histories and death dates that extend beyond the actual time of transplant for these patients. Artificial death dates and status change histories were generated from actual histories of patients in the SRTR database, subject to matching certain patient characteristics. We matched patients on the waitlist for status histories in the following manner: Status 1 patients were only matched to other status 1 patients, and pediatric patients were matched to other pediatric patients. In addition to matching on MELD score, patients were also matched on MELD score slope (positive, 
Table 2

Allocation rules used in LSAM runs. ${ }^{\mathrm{a}}$

\begin{tabular}{lc}
\hline 1999 status-based rules & MELD-based rules \\
\hline Local & Local \\
Status 1 & Status 1 \\
Status 2A & MELD \\
Status 2B & Regional \\
Status 3 & Status 1 \\
Regional & MELD \\
Status 1 & National \\
Status 2A & Status 1 \\
Status 2B & MELD \\
Status 3 & \\
National & \\
Status 1 & \\
Status 2A & \\
Status 2B & \\
Status 3 & \\
\hline
\end{tabular}

${ }^{\text {a }}$ Simulated rules do not include local variances or regional sharing arrangements.

negative, or flat). All runs of the status-based policy used a single medical status input stream, as did all runs of the MELD based policy, but replications differed with respect to Monte Carlo sampling of re-listing probabilities, graft failure times, and organ acceptance probabilities.

\subsection{Policies modeled}

Table 2 lists the allocation policies used in the simulation runs: the 1999 OPTN rule and a hypothesized MELD-based rule. (The listed rules apply to the allocation of livers from adult donors. For a liver from a pediatric donor, the policies further partition each classification into pediatric and adult candidates, with the pediatric subgroup listed first.) The MELD-based rule was defined by aggregating the status $2 \mathrm{~A}$, $2 \mathrm{~B}$, and 3 classifications rule within the local, regional, and national levels. Status 1 was retained as a separate classification, as was the case in the MELD-based policy that was later initiated by the OPTN on February 27, 2002. The simulation runs were performed several months prior to this and were not intended to predict all details of the rule that eventually went into effect.

For the status-based policy, candidates were ordered within each classification in decreasing point order, based on the point award scheme defined in the status-based OPTN policy. (This includes points for wait time rank and points for identity or compatibility of blood type.) For the simulated MELDbased policy, candidates were ordered within each classification in decreasing order of waiting time points (for status 1) or MELD score (for other patients), within geographic distribution area. Candidates were excluded from the match process for criteria defined out in the actual status-based OPTN policy. These criteria were applied to both the MELD-based and the status-based simulated policies. Rank ordering status 1 patients was based on the same system as the status-based policy. As noted earlier, not all details of the 2002 OPTN policy [3] could be anticipated at the time of analysis, but it was
Table 3

Simulated rules versus reality (organs, waitlist, and mortality).

\begin{tabular}{|c|c|c|c|}
\hline Statistic & $\begin{array}{l}\text { Study } \\
\text { cohort }\end{array}$ & $\begin{array}{c}\text { Simulated } \\
1999 \text { status } \\
\text { based } \\
\text { rules }\end{array}$ & $\begin{array}{l}\text { Simulated } \\
\text { MELD } \\
\text { allocation } \\
\text { rules }\end{array}$ \\
\hline \multicolumn{4}{|l|}{ Organs } \\
\hline Transplanted organs & 4482 & 4479 & 4479 \\
\hline Discarded organs & 303 & 309 & 309 \\
\hline \multicolumn{4}{|l|}{ Wait list } \\
\hline Initial waitlist & 11,907 & 11,907 & 11,907 \\
\hline Patient listings & 10,518 & 10,518 & 10,518 \\
\hline Removed from waitlist & & 1504 & 1499 \\
\hline Relistings & 344 & 303 & 316 \\
\hline Retransplants & & 135 & 125 \\
\hline Final waitlist & 14,445 & 14,806 & 15,011 \\
\hline \multicolumn{4}{|l|}{ Mortality } \\
\hline Total deaths & & 2355 & 2114 \\
\hline Wait list deaths & 1806 & 1879 & 1691 \\
\hline Removed patient deaths & & 22 & 25 \\
\hline Relisted patient deaths & & 60 & 61 \\
\hline Post-graft deaths & 353 & 394 & 337 \\
\hline \multicolumn{4}{|l|}{ Mortality by status } \\
\hline Status 1 deaths & 119 & 146 & 151 \\
\hline Status 2A deaths & 172 & 193 & 362 \\
\hline Status 2B deaths & 556 & 564 & 393 \\
\hline Status 3 deaths & 457 & 451 & 306 \\
\hline Inactive status deaths & 502 & 525 & 479 \\
\hline Unknown status deaths & 4 & & \\
\hline \multicolumn{4}{|l|}{ Mortality by meld } \\
\hline MELD 31-40 deaths & & 700 & 555 \\
\hline MELD 21-30 deaths & & 428 & 306 \\
\hline MELD 11-20 deaths & & 344 & 367 \\
\hline MELD 00-12 deaths & & 407 & 463 \\
\hline \multicolumn{4}{|l|}{ Survival } \\
\hline Post-graft surviving & & 3782 & 3826 \\
\hline
\end{tabular}

still possible to simulate a general change to a new system based that orders candidates on the basis of MELD scores.

\subsection{Simulated status-based rules versus reality}

Tables 3 and 4 compare the actual experience of the patients waitlisted in 1999 to the simulated results from LSAM (mean results of ten replications) using the prioritization for organ allocation used in 1999, sorted by status within local, regional, and national. (The number of replications was limited to ten due to long run times.) These results show good agreement for most of the criteria shown, subject to random variation. Extensive sensitivity analyses with various specifications of the model suggest that the results change in expected directions when the inputs are changed. The most notable discrepancy for this model is in the waitlist mortality.

The simulated waitlist mortality is higher than was actually experienced. Changes in the specification of the artificially generated death dates for those patients who received a transplant in real life will lead to better agreement between simulated and actual waitlist mortality. However, it was not possible to make this adjustment without rebuilding the se- 
Table 4

Simulated rules versus reality (transplants).

\begin{tabular}{|c|c|c|c|}
\hline Statistic & $\begin{array}{l}\text { Study } \\
\text { cohort }\end{array}$ & $\begin{array}{c}\text { Simulated } \\
1999 \text { status } \\
\text { based } \\
\text { rules }\end{array}$ & $\begin{array}{l}\text { Simulated } \\
\text { MELD } \\
\text { allocation } \\
\text { rules }\end{array}$ \\
\hline \multicolumn{4}{|l|}{ Transplants by status } \\
\hline Status 1 transplants & 551 & 531 & 525 \\
\hline Status 2A transplants & 931 & 977 & 402 \\
\hline Status 2B transplants & 2541 & 2361 & 1659 \\
\hline Status 3 transplants & 442 & 610 & 1893 \\
\hline \multicolumn{4}{|l|}{ Transplants by meld } \\
\hline MELD 31-40 transplants & & 629 & 813 \\
\hline MELD 21-30 transplants & & 97 & 388 \\
\hline MELD 11-20 transplants & & 820 & 1692 \\
\hline MELD 00-10 transplants & & 2933 & 1586 \\
\hline \multicolumn{4}{|l|}{ Pediatric transplants } \\
\hline Pediatric transplants & 449 & 525 & 667 \\
\hline \multicolumn{4}{|l|}{ Transplants by location } \\
\hline Local transplants & 3298 & 3615 & 3592 \\
\hline Regional transplants & 887 & 689 & 704 \\
\hline National transplants & 282 & 175 & 183 \\
\hline Transplants in region 01 & 133 & 152 & 155 \\
\hline Transplants in region 02 & 630 & 633 & 641 \\
\hline Transplants in region 03 & 697 & 685 & 681 \\
\hline Transplants in region 04 & 403 & 430 & 435 \\
\hline Transplants in region 05 & 675 & 692 & 688 \\
\hline Transplants in region 06 & 135 & 89 & 89 \\
\hline Transplants in region 07 & 463 & 489 & 496 \\
\hline Transplants in region 08 & 331 & 317 & 314 \\
\hline Transplants in region 09 & 313 & 251 & 247 \\
\hline Transplants in region 10 & 312 & 349 & 346 \\
\hline Transplants in region 11 & 375 & 392 & 387 \\
\hline
\end{tabular}

lected MELD histories for all patients. (Such changes can be made in the near future. Our flexibility in changing the specification of the waitlist death rates is currently limited by the need to concurrently change the simulation of MELD scores and status changes for the input data files.) Currently, we have not simulated removal from the waitlist in the artificial patient histories. Inclusion of these removals will also lead to lower waitlist mortality.

\subsection{Simulated status-based rules versus MELD}

Tables 3 and 4 also compare the results of using the 1999 status-based organ allocation rules to the hypothesized MELD allocation rule. Since it was important to include pediatric patients in order to make these results comparable to the status based results, MELD score histories for pediatric patients were selected from adult patients in the University of Michigan study of MELD mortality.

The major differences in allocation with these two sets of rules are as expected. The status based rules lead to more status 1 transplants while the MELD based rule leads to more transplants among patients with high MELD. In addition, the MELD allocation rules lead to lower death rates on the waitlist.

Because of a number of differences (details of the MELD policy and the candidate cohort) exact comparisons to the ac- tual experience of introducing the MELD policy in February 2002 are not to be expected, but it is interesting to observe the general level of change that is predicted. Based on preliminary review of outcomes at six months (February 27, 2002 through August 27, 2002), deaths among patients on the waitlist decreased by $23 \%$, after adjusting for changes in the size of the waitlist the year before [17]. This compares to a $10 \%$ reduction for the simulated results. When more data from the actual introduction of a MELD-based rule become available, the analysis presented here can be repeated with data for the 2002 cohort and with simulated policy approximating that actually implemented.

\section{Conclusion}

In its first application, the LSAM prototype was quickly adapted to support a prospective investigation of the transition to a new liver allocation policy. The model reproduced important features of historically observed allocations and outcomes, and it predicted changes in the directions anticipated for a hypothetical policy based on the use of MELD and PELD scores. Subsequently, LSAM is undergoing further review and refinement, and the other simulations, TSAM and KPSAM, are being readied for analyses of heart, lung, kidney, and pancreas allocation policies. One of the refinements in development is a facility to generate synthetic data for patient and organ arrival streams and for patient status histories. This facility will help model users explore a range of assumptions about the future incidence of end-stage liver disease and the availability of donor organs, and the implications of these assumptions for outcomes like life years saved. The power and flexibility demonstrated in this initial experience suggest that our modeling approach will prove useful in analyzing a wide variety of organ allocation policies and related issues of importance to the transplantation community.

\section{Acknowledgements}

This work is supported by the Scientific Registry of Transplant Recipients (contract number 231-00-0116), with funding and oversight by the Health Resources and Services Administration, a division of the U.S. Department of Health and Human Services.

\section{References}

[1] Scientific Registry of Transplant Recipients, Quick Facts (University Renal Research and Education Association, Ann Arbor, MI, 2002).

[2] University Renal Research and Education Association, United network for organ sharing, Annual report of the U.S. organ procurement and transplantation network and the scientific registry of transplant recipients: Transplant data 1992-2001, Department of Health and Human Services, Health Resources and Services Administration, Office of Special Programs, Division of Transplantation, Rockville, MD (2003). 
[3] United Network for Organ Sharing, Allocation of Livers, Proposed Amended UNOS Policy 3.6, Approved by OPTN/UNOS Board of Governors, November 15, 2001, revised February 1, 2002 Richmond, VA (2002).

[4] D.H. Howard, Dynamic analysis of liver allocation policies, Medical Decision Making 21 (2001) 257-266.

[5] J. Ratliffe, T. Young, M. Buxton, T. Eldabi, R. Paul, A. Burroughs, G. Papatheodoridis and K. Rolles, A simulation modeling approach to evaluating alternative policies for the management of the waiting list for liver transplantation, Health Care Management Science 4 (2001) $117-124$.

[6] A.M. Harper, S.E. Taranto, E.B. Edwards and O.P. Daily, An update on a successful simulation project: The UNOS liver allocation model, in: Proceedings of the 2000 Winter Simulation Conference, Piscataway, NJ (Institute of Electrical and Electronics Engineers, NJ, 2000).

[7] A.A.B. Pritsker, Life and death decisions: Organ transplantation allocation policy analysis, OR/MS Today, August (1998).

[8] A.A.B. Pritsker et al., Organ transplantation policy evaluation, in: Proceedings of the 1995 Winter Simulation Conference, eds. C. Alexopoulos, K. Kang, W. Lilegdon and D. Goldsman (Institute of Electrical and Electronics Engineers, Piscataway, NJ, 1995).

[9] A.A.B. Pritsker, O.P. Daily and K.D. Pritsker, Using simulation to craft a national organ transplantation policy, in: Proceedings, 1996 Western Multiconference of the Society for Computer Simulation - Simulation in the Medical Sciences (The Society for Computer Simulation, San Diego, 1996).

[10] A.A.B. Pritsker et al., Organ transplantation modeling and analysis, in: Proceedings of the 1996 Winter Simulation Conference, Piscat- away, NJ, eds. J.M. Charnes, D.J. Morrice, D.T. Brunner and J.J. Swain (Institute of Electrical and Electronics Engineers, Piscataway, NJ, 1996).

[11] United Network for Organ Sharing, Report of the UNOS Distribution Committee to the UNOS Liver and Intestinal Organ Transplantation Committee, Richmond, VA (2000).

[12] University Renal Research and Education Association, User Manual for LSAM, Version 1.2 (Ann Arbor, MI, 2002).

[13] R.N.H. Pugh, I.M. Murray-Lyon, J.L. Dawson, M.C. Pictioni and R. Williams, Transection of the oesophagus for bleeding oesophageal varices, British Journal of Surgery 60 (1973) 646-649.

[14] P.S. Kamath, R.H. Wiesner, M. Malinchoc, W. Kermers, T.M. Therneau, C.L. Kosberg, G. D'Amico, E.R. Dickson and W.R. Kim, A model to predict survival in patients with end-state liver disease, $\mathrm{He}$ patology 33 (2001) 464-470.

[15] R.M. Merion, R.A. Wolfe, D.M. Dykstra, A.B. Leichtman, B. Gillespie and P.J. Held, Longitudinal assessment of mortality risk among candidates for liver transplantation, Liver Transplantation 9 (2003) 12-18.

[16] S.V. McDiarmid, R. Anand, A.S. Lindblad and the Principal Investigators and Institutions of the Studies of Pediatric Liver Transplantation (SPLIT) Research Group, Development of a pediatric end-stage liver disease score to predict poor outcome in children awaiting liver transplantation, Transplantation 74 (2002) 173-181.

[17] United Network for Organ Sharing, Liver policy outcomes encouraging at six months; some adjustments recommended (Press Release, Richmond, VA, November 13, 2002). 